\title{
Expression of, and in vivo stressosome formation by, single members of the RsbR protein family in Bacillus subtilis
}

\author{
Correspondence \\ William Haldenwang \\ Haldenwang@uthscsa.edu
}

Received 2 November 2009

Revised 11 December 2009

Accepted 16 December 2009

\author{
Adam Reeves, Luis Martinez and William Haldenwang
}

Department of Microbiology and Immunology, The University of Texas Health Science Center at San Antonio, San Antonio, TX 78229-3900, USA

\begin{abstract}
The Bacillus subtilis stressosome is a $1.8 \mathrm{MDa}$ complex that is the focal point for activating the bacterium's general response to physical stress. In vitro studies demonstrated that the stressosome's core element can be formed from one or more of a family of paralogous proteins (RsbRA, -RB, -RC and -RD) onto which the system's activator protein (RsbT) and its principal inhibitor (RsbS) are bound. The RsbR components of the stressosome are envisioned to be the initial receptors of stress signalling with the stressosome structure itself serving as a device to integrate multiple stress signals for a coordinated response. In the current work, we examine several of the in vivo characteristics of the RsbR family members, including their expression and ability to form stressosomes to regulate $\sigma^{\mathrm{B}}$. Translational fusions of lac $Z$ to each $r s b R$ paralogue revealed that $r s b R A,-R B$ and $-R C$ are expressed at similar levels, which remain relatively constant during growth, ethanol stress and entry into stationary phase. $r s b R D$, in contrast, is expressed at a level that is only slightly above background during growth, but is induced to $30 \%$ of the $r s b R$ expression level following ethanol stress. Velocity sedimentation analyses of $B$. subtilis extracts from strains expressing single $r s b R$ paralogues demonstrated that each incorporates RsbS into fast-sedimenting complexes. However, consistent with rsbRD's lower expression, the RsbRDdependent RsbS complexes were present at only $20 \%$ of the level of the complexes seen in a wild-type strain. The lower stressosome level in the RsbRD strain is still able to hold RsbT's activity in check, implying that the RsbR/S component of stressosomes is normally in excess for the control of RsbT. Consistent with such a notion, reporter gene and Western blot assays demonstrate that although RsbT is synthesized at the same rate as RsbRA and RsbS, RsbT's ultimate level in growing $B$. subtilis is only $10 \%$ that of RsbRA. Apparently, RsbT's inherent structure and/or its passage between the stressosome and its activation target compromises its persistence.
\end{abstract}

\section{INTRODUCTION}

Bacillus subtilis relies on an alternative sigma factor $\left(\sigma^{\mathrm{B}}\right)$ to respond to environmental or nutritional stress (reviewed by Hecker et al., 1996, 2007). $\sigma^{\mathrm{B}}$ controls the general stress regulon, a collection of over 150 genes whose products confer resistance to prolonged starvation or physical damage (Hecker et al., 1996, 2007; Petersohn et al., 1999; Price et al. 2001). In the absence of stress, $\sigma^{\mathrm{B}}$ is inactive, bound to the anti- $\sigma^{\mathrm{B}}$ protein RsbW (Benson \& Haldenwang, 1993). The release of $\sigma^{\mathrm{B}}$ from RsbW is triggered by the binding of an additional protein (RsbV) to RsbW (Delumeau et al., 2002; Dufour \& Haldenwang, 1994). In unstressed B. subtilis, $\mathrm{RsbV}$ is unable to effect the release of $\sigma^{\mathrm{B}}$ due to an RsbWdependent phosphorylation (Dufour \& Haldenwang, 1994). Dephosphorylation and reactivation of RsbV occurs by

Abbreviations: LB, Luria-Bertani; $\sigma^{\mathrm{B}}$, sigma B. either of two stress-responsive phosphatases (RsbP and RsbU) (Kang et al., 1996; Vijay et al., 2000; Voelker et al., 1995; Yang et al., 1996). The RsbP phosphatase responds to nutritional stress (e.g. glucose or phosphate limitation) and requires an additional protein (RsbQ: an $\alpha / \beta$ hydrolase) for its activity (Brody et al., 2001; Delumeau et al., 2002; Vijay et al., 2000). The actual inducer of RsbP/Q phosphatase activity is unknown, but RsbP/Q activation occurs coincident with a drop in cellular ATP levels (Voelker et al., 1996; Zhang \& Haldenwang, 2005). RsbU, the phosphatase reactive to physical stress (e.g. ethanol, osmotic or heat shock), also requires another protein (RsbT) for activity (Yang et al., 1996). In the absence of stress, RsbT is believed to be bound to its primary negative regulator (RsbS) and a family of homologous proteins RsbR (RsbRA), YkoB (RsbRB), YojH (RsbRC) and YqhA (RsbRD) in a large $(1.8 \mathrm{MDa})$ complex termed the 'stressosome' (Akbar et al., 1997, 2001; Chen et al., 2003; Delumeau et al., 2006; Kim 
et al., 2004b; Marles-Wright et al., 2008). rsbRA is cotranscribed with the $\sigma^{\mathrm{B}}$ structural gene and the other key $\sigma^{\mathrm{B}}$ regulators, while the $r s b R$ paralogues are expressed from diverse sites along the B. subtilis chromosome (Akbar et al., 2001). Exposure to physical stress is thought to induce an RsbT-dependent phosphorylation of both RsbR and RsbS (Chen et al., 2003, Gaidenko et al., 1999; Yang et al., 1996). This allows the release of RsbT from the complex and its activation of RsbU (Hardwick et al., 2007). The phosphorylation of RsbR facilitates the phosphorylation of RsbS (Chen et al., 2003, 2004; Gaidenko et al., 1999), suggesting a sequence of events in which RsbR phosphorylation precedes the phosphorylation of RsbS. There is a fifth RsbR paralogue (YtvA) that lacks the RsbT-dependent phosphorylation sites found in the other RsbR paralogues and instead carries an $\mathrm{N}$-terminal light, oxygen or voltage domain (Avila-Perez et al., 2006; Gaidenko et al., 2006). Although unlikely to be regulated like the other RsbR paralogues, YtvA has been observed to cofractionate with stressosomes and enhance $\sigma^{\mathrm{B}}$ activity in the presence of blue light (Gaidenko et al., 2006; Suzuki et al., 2007). The RsbR proteins are hypothesized to be the initial targets for stress signalling, with stressgenerated changes allowing their phosphorylation as the prerequisite for RsbS phosphorylation and the release of RsbT (Eymann et al., 2007; Kim et al., 2004a; Yang et al., 1996). The activation of $\sigma^{\mathrm{B}}$ is transient. The system is reset to pre-stress levels by an additional phosphatase (RsbX) that dephosphorylates RsbR-P and RsbS-P, reactivating their ability to capture RsbT (Chen et al., 2004; Delumeau et al., 2006; Yang et al., 1996). The regulation of RsbX's activity is unclear. A $\sigma^{\mathrm{B}}$-dependent promoter increases expression of $r s b X$ following $\sigma^{\mathrm{B}}$ activation (Dufour et al., 1996); however, the absolute levels of RsbX may not be the sole factor modulating its activity. Although elimination of RsbX results in a severe growth defect due to high $\sigma^{\mathrm{B}}$ activity, manipulation of RsbX levels from an inducible promoter does not alter the stress inducibility of $\sigma^{\mathrm{B}}$ (Voelker et al., 1997).

Structural studies of the B. subtilis stressosome portray a complex formed from multiple RsbR proteins with the RsbR C-terminal regions forming a base upon which RsbS and RsbT rest (Marles-Wright et al., 2008). In this model, the RsbR $\mathrm{NH}_{2}$-termini project outward as possible sensory extensions (Marles-Wright et al., 2008). Although this work employed RsbRA as the RsbR source, it is likely that the other RsbRs can form similar structures. The Cterminal portion of RsbR, which forms the stressosome base, is highly conserved among the paralogues and each RsbR variant can form a high molecular mass structure that incorporates RsbS in vitro (Delumeau et al., 2006; Kim et al., 2004b). RsbRB and RsbRC proteins have also been detected in anti-RsbRA immunoprecipitates from stressosome-containing fractions, suggesting their presence in a common complex (Delumeau et al., 2006; Kim et al., 2004b). The explicit functions of the multiple RsbR proteins in B. subtilis are unclear. They could serve as responders to unique stress signals; however, if this is so, their responsiveness is overlapping. The loss of one or several of the RsbR paralogues does not prevent $B$. subtilis from responding to the environmental stresses that normally activate $\sigma^{\mathrm{B}}$. Only when all of the RsbR family members are deleted does $\sigma^{\mathrm{B}}$ become largely unregulated (Akbar et al., 2001). Multiple $r s b R$ genes might also represent a device to maintain adequate levels of RsbR under changing environmental conditions. Each $r s b R$ could be differentially expressed so as to ensure a source of RsbR regardless of growth state.

In the current work, we examine several in vivo properties of the RsbR family of proteins, including their expression, and ability to form stressosomes and regulate $\sigma^{\mathrm{B}}$. Translational fusions of $l a c Z$ to each $r s b R$ gene revealed that $r s b R A,-R B$ and $-R C$ are expressed at similar levels, which remain relatively constant during growth and stress. $r s b R D$, in contrast, is expressed at lower levels and is stress inducible. Velocity sedimentation analysis of $B$. subtilis extracts from strains expressing a single $r s b R$ paralogue demonstrated that each forms fast-sedimenting complexes that incorporate RsbS. The RsbRD-dependent complexes were present at only $20 \%$ of the level seen in a wild-type strain and yet were able to control $\sigma^{\mathrm{B}}$, implying that RsbR and $-S$ are normally in excess for the control of RsbT. Consistent with such a notion, reporter gene and Western blot assays indicate that the RsbT level in growing B. subtilis is only $10 \%$ that of RsbR.

\section{METHODS}

Bacterial strains and plasmids. All strains and their relevant genotypes are listed in Table 1. B. subtilis strains are derivatives of PY22 (Benson \& Haldenwang, 1992). Standard recombinant DNA methods were used as described by Sambrook et al. (1989) with Escherichia coli $\mathrm{DH} 5 \alpha$ as the host for all cloning. To delete selective $r s b R$ paralogues, PY22 was transformed with chromoso-

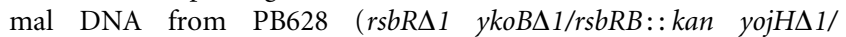
rsbRC::erm yqhA $1 / r s b R D:: s p c$ ) (Akbar et al., 2001). The initial transformants were selected for $\operatorname{Erm}^{\mathrm{R}}$ creating BSH143 (yojH $\Delta 1$ ) rsbRC::erm). BSH143 was then transformed to SP $\beta$ ctc::lacZ $\left(\right.$ Tet $^{\mathrm{R}}$, Erm $^{\mathrm{R}}$ ) using chromosomal DNA from BSH80 (Reeves et al., 2007) and $\mathrm{Tet}^{\mathrm{R}}$ selection to create BSH151. BSH151 was subsequently transformed with PB628 DNA selecting for $\mathrm{Spc}^{\mathrm{R}}$, yielding BSH153 (yojH $\Delta 1 / r s b R C::$ erm $\left.y q h A \Delta 1 / r s b R D:: s p c\right)$. Transformation of BSH153 with PB628 chromosomal DNA and

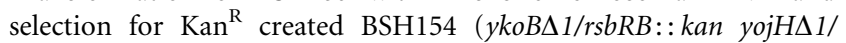
rsbRC::erm $y q h A \Delta 1 / r s b R D:: s p c)$. BSH144 (ykoBAl/rsbRB::kan $y q h A \Delta 1 / r s b R D:: s p c)$ is BSH80 transformed sequentially to $\mathrm{Kan}^{\mathrm{R}}$ and $\mathrm{Spc}^{\mathrm{R}}$ with PB628 DNA. During the transformation of BSH153 with PB628 DNA, dark blue colonies appeared at a low frequency. These were probably strains in which the final $r s b R$ gene ( $r s b R A)$ had been lost by the simultaneous uptake of the $r s b R \Delta 1$ mutation with $y k o B \Delta 1 r s b R B:: k a n$. The $r s b R \Delta 1$ deletion mutation was verified by PCR. A representative strain lacking all of the $r s b R$ paralogues genes was designated BSH155. BSH214 was created by placing a $\mathrm{Cm}^{\mathrm{R}}$ cassette upstream of the $\operatorname{sigB}$ operon by transforming BSH155 with linearized pARE241 (described below) and $\mathrm{Cm}^{\mathrm{R}}$ selection.

A plasmid (pARE241) was constructed to link an antibiotic resistance cassette to an $r s b R A$ deletion. To construct this plasmid, a $1 \mathrm{~kb}$ DNA fragment extending upstream from the third codon of $r s b R A$ was 
Table 1. Strains and plasmids used in this study

\begin{tabular}{|c|c|}
\hline Strain or plasmid & Relevant genotype or feature \\
\hline \multicolumn{2}{|l|}{ Plasmids } \\
\hline pUC19 & $\mathrm{Ap}^{\mathrm{r}}$ \\
\hline pJM102/pJM103 & $\mathrm{Ap}^{\mathrm{r}} \mathrm{Cm}^{\mathrm{r}}$ \\
\hline pSGMU37 & $\mathrm{Ap}^{\mathrm{r}} \mathrm{Cm}^{\mathrm{r}}$ \\
\hline pARE7 & $\mathrm{Ap}^{\mathrm{r}} \operatorname{Kan}^{\mathrm{r}} P_{A} r s b R r s b S r s b T r s b U$ \\
\hline pLAM1 & $\begin{array}{l}\mathrm{Ap}^{\mathrm{r}} \mathrm{Cm}^{\mathrm{r}} 1 \mathrm{~kb} \text { upstream-1 } \mathrm{kb} \\
\text { downstream of } y k o B\end{array}$ \\
\hline pARE141 & $\mathrm{Ap}^{\mathrm{r}} 1 \mathrm{~kb}$ upstream of $\operatorname{sig} B$ operon $\left(P_{A}\right)$ \\
\hline pARE212 & $\mathrm{Ap}^{\mathrm{r}} \mathrm{Cm}^{\mathrm{r}} \mathrm{Cm}$ cassette upstream of $\operatorname{sig} B$ \\
\hline pARE236 & $\mathrm{Ap}^{\mathrm{r}} \mathrm{Cm}^{\mathrm{r}} y k o B:: l a c Z$ \\
\hline pARE237 & $\mathrm{Ap}^{\mathrm{r}} \mathrm{Cm}^{\mathrm{r}} y q h A:: l a c Z$ \\
\hline pARE238 & $\mathrm{Ap}^{\mathrm{r}} \mathrm{Cm}^{\mathrm{r}} \mathrm{rsbR}:: l a c Z$ \\
\hline pARE241 & $\mathrm{Ap}^{\mathrm{r}} \mathrm{Cm}^{\mathrm{r}} P_{A} r s b S r s b T$ \\
\hline pARE244 & $\mathrm{Ap}^{\mathrm{r}} \mathrm{Cm}^{\mathrm{r}} P_{A} y q h A r s b S r s b T$ \\
\hline pARE269 & $\mathrm{Ap}^{\mathrm{r}} \mathrm{Cm}^{\mathrm{r}} \mathrm{rsbS}:: \operatorname{lac} Z$ \\
\hline pARE270 & $\mathrm{Ap}^{\mathrm{r}} \mathrm{Cm}^{\mathrm{r}} r s b T:: l a c Z$ \\
\hline pARE278 & $\mathrm{Ap}^{\mathrm{r}} \mathrm{Cm}^{\mathrm{r}}$ yojH::lacZ \\
\hline pARE282 & $\mathrm{Ap}^{\mathrm{r}} \mathrm{Cm}^{\mathrm{r}} P_{A}:: k a n:: r s b S r s b T$ \\
\hline \multicolumn{2}{|c|}{ B. subtilis strains } \\
\hline PY22 & Wild-type \\
\hline BSH80 & SP $\beta$ ctc: : lac $Z$ \\
\hline PB628 & $\begin{array}{l}r s b R \Delta 1 \text { ykoB } \Delta 1:: \text { kan yojH } \Delta 1:: \text { ery } \\
y q h A \Delta 1:: s p c\end{array}$ \\
\hline BSH143 & yojH $\Delta 1:$ : ery \\
\hline BSH144 & $\begin{array}{l}y k o B \Delta 1:: \text { kan } y q h A \Delta 1:: s p c \\
\operatorname{SP} \beta \text { ctc: : lac } Z\end{array}$ \\
\hline BSH151 & yojH $\Delta 1::$ ery $\mathrm{SP} \beta$ ctc: : lacZ \\
\hline BSH153 & yojH $\Delta 1:$ : ery yqhA $\Delta 1::$ spc SP $\beta$ ctc: : lac $Z$ \\
\hline BSH154 & $\begin{array}{l}y k o B \Delta 1:: \text { kan yojH } \Delta 1:: \text { ery yqh } A \Delta 1:: \operatorname{spc} \mathrm{SP} \beta \\
\text { ctc: : lacZ }\end{array}$ \\
\hline BSH155 & $\begin{array}{l}r s b R \Delta 1 \text { ykoB } \Delta 1:: \text { kan yojH } \Delta 1:: \text { ery } \\
y q h A \Delta 1:: \operatorname{spc} \operatorname{SP} \beta \text { ctc: : lacZ }\end{array}$ \\
\hline BSH214 & $\begin{array}{l}\text { rsbR } \Delta 1(\mathrm{Cm}) \text { ykoB } 1:: \text { kan yojH } \Delta 1: \text { : ery } \\
y q h A \Delta 1:: \text { spc SP } \beta \text { ctc:: lac } Z\end{array}$ \\
\hline BSA177 & $y k o B:: \operatorname{lac} Z$ \\
\hline BSA178 & yojH: : lacZ \\
\hline BAR179 & $y q h A:: l a c Z$ \\
\hline BAR180 & $r s b R:: l a c Z$ \\
\hline BAR199 & 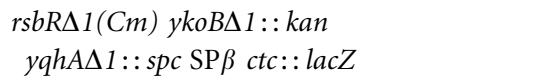 \\
\hline BAR203 & rsbR $\Delta 1(\mathrm{Cm})$ yojH $\Delta 1::$ ery $\mathrm{SP} \beta$ ctc: : lacZ \\
\hline BAR204 & $\begin{array}{l}r s b R \Delta 1(\mathrm{Cm}) \text { yojH} H 1:: \text { ery yqhA } \Delta 1:: \operatorname{spc} \mathrm{SP} \beta \\
\quad c t c:: \text { lacZ }\end{array}$ \\
\hline BAR205 & 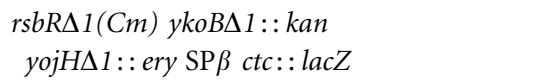 \\
\hline BAR230 & $y k o B \Delta 2$ \\
\hline BAR231 & ykoB $\Delta 2$ yojH $\Delta 1::$ ery \\
\hline BAR236 & $y k o B \Delta 2$ yojH $\Delta 1::$ ery $\mathrm{SP} \beta$ ctc: : lacZ \\
\hline BAR250 & $r s b S:: l a c Z$ \\
\hline BAR251 & $r s b T:: l a c Z$ \\
\hline BAR298 & $\begin{array}{l}y k o B \Delta 2 \text { yojH } \Delta 1:: \text { ery yqhA } \Delta 1:: \text { spc SP } \beta \\
\text { ctc:: lacZ }\end{array}$ \\
\hline BAR299 & $\begin{array}{l}\Delta r s b R:: \text { kan ykoB } \Delta 2 \text { yojH } \Delta 1:: \text { ery } \\
y q h A \Delta 1:: \text { spc SP } \beta \text { ctc:: lac } Z\end{array}$ \\
\hline BAR308 & $\begin{array}{l}P_{A} y q h A \text { ykoB } \Delta 2 \text { yojH } \Delta 1:: \text { ery } \\
y q h A \Delta 1:: \text { spc SP } \beta \text { ctc: : lacZ }\end{array}$ \\
\hline
\end{tabular}

amplified from PY22 chromosomal DNA using oligonucleotide primers that added Bam $\mathrm{H}$ and SalI restriction endonuclease sites at its ends. The resulting fragment was cut with BamH and SalI and cloned into similarly cut pUC19 to create pARE141. pARE141 was then linearized at a unique ClaI site within the B. subtilis sequence $300 \mathrm{bp}$ upstream of the rsbRA initiation codon and a $\mathrm{Cm}$ cassette [amplified from pJM102 (Jonas et al., 1990)] was cloned into this site, creating pARE212. A SalI-SphI fragment extending from the third codon before the end of $r s b R A$ for approximately $1 \mathrm{~kb}$ to the termination codon of $r s b T$ was similarly amplified from PY22 DNA and cloned into the corresponding sites in pARE212, creating pARE241. pARE241 carries an in-frame deletion of $r s b R A(r s b R A \Delta 2)$ linked to $\mathrm{Cm}^{\mathrm{R}}$ within a $B$. subtilis DNA element. pARE241 was

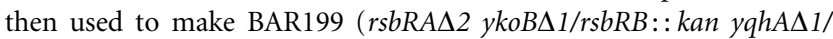
$r s b R D:: s p c)$ following transformation of BSH144 ( $y k o B \Delta 1$ / $r s b R B::$ kan $y q h A \Delta 1 / r s b R D:: s p c)$ with linearized pARE241. Colonies selected on the basis of $\mathrm{Cm}^{\mathrm{R}}$ were screened by Western blot for those lacking RsbRA, resulting in a strain expressing only $y o j H / r s b R C$ as its source of RsbR. A strain (BAR204) expressing only $y k o B / r s b R B$ was similarly constructed by transforming BSH151 (yojHAl/rsbRC: : erm) with pARE241 to introduce the $r s b R A \Delta 2$ mutation (BAR203) followed by transformation with BSH155 chromosomal DNA and selection for $\mathrm{Spc}^{\mathrm{R}}(y q h A \Delta 1 / r s b R D:: s p c)$. Similarly, to create a strain that only expressed $y q h A / r s b R D$, BAR203 was transformed with $\mathrm{BSH} 155$ chromosomal DNA with selection for $\operatorname{Kan}^{\mathrm{R}}(y k o B \Delta 1)$ rsbRB:: kan) colonies.

Other strains missing some or all of the RsbR paralogues were created in a $y k o B / r s b R B$ deletion strain. The parent strain was constructed using a plasmid (pLAM1) containing DNA elements upstream and downstream of $y k o B / r s b R B$. pLAM1 consists of an EcoRI-PstI fragment extending from $1 \mathrm{~kb}$ upstream of $y k o B / r s b R B$ to the $y k o B / r s b R B$ start codon, joined to a PstI-HindIII fragment extending $1 \mathrm{~kb}$ downstream from the stop codon of $y k o B / r s b R B$ cloned into pJM102. pLAM1 was used to delete the $y k o B / r s b R B$ coding sequence by first transforming PY22 using the plasmidencoded $\mathrm{Cm}^{\mathrm{R}}$ to select for integration of the plasmid at $y \mathrm{koB} / \mathrm{rs} b \mathrm{RB}$. $\mathrm{Cm}^{\mathrm{R}}$ clones were passaged in the absence of chloramphenicol and replica-plated to identify $\mathrm{Cm}$-sensitive colonies. These were expected to include clones where the plasmid had recombined out of the chromosome. Half of such plasmid excisions would remove the wild-type copy of $y k o B / r s b R B$, leaving the deletion in its place. Unique primers external to $y k o B$ were used to verify the presence of the $y k o B / r s b R B$ deletion by PCR. A representative clone was designated BAR230. BAR231 ( $y k o B \Delta 2 / r s b R B$ yojH $1 /$ rsbRC: : erm) was created by transforming BAR230 with BSH143, selecting for $\mathrm{Erm}^{\mathrm{R}}$. The $c t c$ : : lacZ reporter was then inserted to create BAR236 by transforming BAR231 with BSH80 chromosomal DNA and selecting

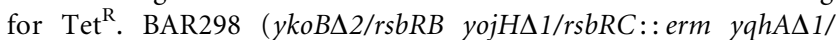
$r s b R D:: s p c$ SP $\beta$ ctc::lacZ), a strain only expressing $r s b R A$, was created by transforming BAR236 ( $y k o B \Delta 2 / r s b R B$ yojH $\Delta 1$ ) rsbRC::erm SP $\beta$ ctc::lacZ) with PB628 chromosomal DNA and selecting for $\mathrm{Spc}^{\mathrm{R}}(y q h A \Delta 1 / r s b R D:: s p c)$.

To insert $y q h A / r s b R D$ into the $\operatorname{sig} B$ operon in lieu of $r s b R A$, a strain was first constructed in which a $\operatorname{Kan}^{\mathrm{R}}$ cassette (Guérout-Fleury et al., 1995) was placed within the ORF of $r s b R A$. To do this, a $B a m \mathrm{HI}-\mathrm{Xba \textrm {I }}$ fragment extending $1 \mathrm{~kb}$ upstream of $r s b R$ to the 117 th bp of the $r s b R$ ORF was cloned into pJM103. Next, a SalI$S p h$ I fragment starting 9 bp upstream of the $r s b R$ termination codon and extending to the $r s b T$ termination codon was joined to the previous construct. A $\mathrm{Kan}^{\mathrm{R}}$ cassette was then cloned into the $X b a \mathrm{I}-$ SalI sites between the two B. subtilis DNA fragments, creating pARE282. A strain BAR299 ( $\triangle r s b R A:$ : kan $y k o B \Delta 2 / r s b R B$

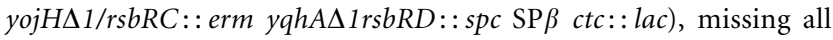
of the RsbR paralogues, was then constructed by transforming BAR298 $(y k o B \Delta 2 / r s b R B$ yojH $1 / r s b R C::$ erm $y q h A \Delta 1 r s b R D:: s p c$ 
SP $\beta$ ctc: : lac) with ScaI-linearized pARE282 and selecting for $\mathrm{Kan}^{\mathrm{R}}$, screening for dark blue $\mathrm{Lac}^{+}$colonies indicative of high $\sigma^{\mathrm{B}}$ activity and the absence of RsbR by Western blot of clone extracts. BAR308 $\left(P_{A} \quad y q h A / r s b R D \quad \Delta y k o B / r s b R B\right.$ yojH $\Delta 1 / r s b R C::$ erm $y q h A \Delta 1:: s p c$ SP $\beta$ ctc: : lacZ) was created by transforming BAR299 with linearized pARE244, a plasmid formed by placing a $y q h A / r s b R D$ gene fragment extending from the gene's initiation codon to its terminal codon in the correct orientation into pARE241. The oligonucleotides used in the PCR amplification provided appropriate restriction endonuclease sites (SalI) to allow the placement of the $y q h \mathrm{~A} /$ $r s b R D$ ORF into the plasmid-encoded $\operatorname{sig} B$ operon as a translational fusion to the rsbRA initiation codon. The incorporation of the sequences for the restriction endonuclease sites resulted in an RsbRD coding sequence with Met-Ser-Thr and Val-Asp-Leu-GlyGlu added to its $\mathrm{N}$ - and C-terminus, respectively. B. subtilis transformed with pARE244 were isolated on the basis of $\mathrm{Cm}^{\mathrm{R}}$ and screened for Kan sensitivity and a reduction in the blue colony $\left(\mathrm{RsbR}^{-}\right)$phenotype.

To obtain B. subtilis strains in which $y k o B / r s b R B$ (BSA177), yojH/ $r s b R C$ (BSA178), yqha/rsbRD (BAR179) and $r s b R A$ (BAR180) are fused to lac $Z$, DNA segments extending from $1 \mathrm{~kb}$ upstream to the 207 th bp of $y k o B$, the 44 th bp of $y o j H$, the 57 th bp of $y q h A$ and the 23rd bp of $r s b R A$ were amplified by PCR from PY22. The resulting DNAs were cloned into the plasmid vector pSGMU37 (Errington, 1986) to create pARE278, pARE236, pARE237 and pARE238, plasmids encoding translational fusions of E. coli lac $Z$ to $y k o B$, $y o j H, y q h A$ and $r s b R A$, respectively. The lac $Z$ chimeras were placed into their homologous $r s b R$ loci within wild-type B. subtilis (PY22) to create BSA177-180 by transformation and selection for plasmid integration on the basis of $\mathrm{Cm}^{\mathrm{R}}$. Translational fusions of $r s b S$ and $r s b T$ to $l a c Z$ were made by amplifying DNA fragments from PY22, starting at the 358 th bp of the $822 \mathrm{bp} r s b R$ gene and extending to either the 37th bp of $r s b S$ or the 17th bp of $r s b T$. Cloning of each of the two fragments into pSGMU37 created translational fusions of $r s b S$ (pARE269) and $r s b T$ (pARE270) to lacZ. pARE269 and pARE270 were integrated into the sigB operon of B. subtilis (PY22) by transformation and $\mathrm{Cm}^{\mathrm{R}}$ selection to isolate BSA250 and 251, respectively.

Velocity sedimentation analysis. $B$. subtilis was grown to $\mathrm{OD}_{540}$ 0.7 in Luria-Bertani (LB) medium (Sambrook et al., 1989), harvested

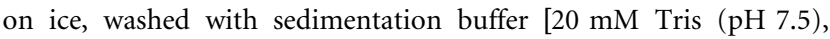
$10 \mathrm{mM} \mathrm{MgCl} 2,0.5 \mathrm{mM}$ EDTA, $1 \mathrm{mM}$ DTT, $0.1 \mathrm{M} \mathrm{NH}_{4} \mathrm{Cl}, 0.03 \%$ PMSF], resuspended in $1 / 20$ vol. of sedimentation buffer and disrupted by passage (twice) through a French pressure cell. Debris was removed by low-speed centrifugation $(8000 \mathrm{~g}, 15 \mathrm{~min})$. A $200 \mu \mathrm{l}$

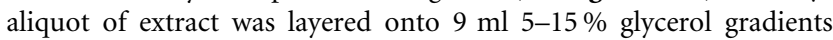
prepared in sedimentation buffer and centrifuged (35000 r.p.m.) in a Beckman SW41 rotor for $6 \mathrm{~h}$ at $4{ }^{\circ} \mathrm{C}$. Fractions of $0.6 \mathrm{ml}$ were collected from the gradients, precipitated by 2 vols ethanol and analysed by SDS-PAGE and Western blot. Western blot images were captured and the intensity of the RsbR and RsbS bands were quantified using a Kodak EDS 290 gel picture station and Kodak 1D.v.3.6.3 software.

General methods. B. subtilis was grown for $\beta$-galactosidase assays with shaking in LB medium and harvested at the OD values indicated in the tables. When the effects of physical stress were to be measured, ethanol ( $4 \%$ final concentration) was added to exponentially growing cultures. $\beta$-Galactosidase assays were performed with chloroform-permeabilized cells, as described by Kenney \& Moran (1987). Western blot assays were performed as previously described with mouse monoclonal antibodies against RsbR and RsbS (Dufour et al., 1996). B. subtilis transformation was carried out by using the method described by Yasbin et al. (1973).

\section{RESULTS AND DISCUSSION}

\section{Expression patterns of rsbRA and its paralogues}

The functions of each of the RsbR paralogues appear to be at least partially redundant with the loss of any of them having little or no consequence on $\sigma^{\mathrm{B}}$ regulation (Akbar et al., 2001). A possible role for the RsbRs in the face of this apparent redundancy could involve their alternative expression under particular growth or stress conditions as a way of ensuring a constant supply of RsbR-like proteins within the cell. To explore whether the rsbRs might be differentially expressed, we created translational fusions between each of their coding sequences and the $E$. coli lac $Z$ gene. The resulting chimeras were integrated into the B. subtilis chromosome at the site of each of the $r s b R$ genes, placing $\beta$-galactosidase expression under the control of the transcriptional and translational elements of each $r s b R$. Analysis of the $\beta$-galactosidase levels in the resulting $B$. subtilis strains (Table 2) revealed that the expression levels of $y k o B(r s b R B)$ and $y o j H(r s b R C)$ are comparable $(\sim 80 \%)$ to that of $r s b R A$ during growth and, like $r s b R A$ expression, are relatively unchanged during exposure to ethanol stress or entry into stationary phase. $y q h A(r s b R D)$, in contrast, is expressed at a level that is only slightly higher than background during growth, but is induced to $30 \%$ of the $r s b R A$ expression level following ethanol stress. The increase in $r s b R D$ expression following stress is consistent with a previous report that $y q h A(r s b R D)$ is among a group of genes that could be identified as $\sigma^{\mathrm{B}}$ regulated in a promoter consensus-directed search (Petersohn et al., 1999). LacZ levels similar to those seen following ethanol stress were obtained following exposure of each of the lac $Z$ fusion strains to salt shock or treatment with the cell-wall antibiotic bacitracin, i.e. $r s b R A,-R B$ and $-R C$ were unaffected, while $r s b R D$ was modestly induced (data not shown). Overall, there is no obvious difference in the expression of RsbRA, -RB and -RC under the conditions

Table 2. Expression of $r s b R$ genes during growth and stress

\begin{tabular}{|c|c|c|c|}
\hline \multirow[t]{2}{*}{ Strain } & \multirow[b]{2}{*}{$\begin{array}{c}\text { Growth } \mathrm{OD}_{540}, \\
0.2-0.4\end{array}$} & \multicolumn{2}{|c|}{$\begin{array}{c}\beta \text {-Galactosidase } \\
\text { activity }^{\star} \text { (Miller units) }\end{array}$} \\
\hline & & $\begin{array}{c}\text { Ethanol } \\
(4 \%)\end{array}$ & $\begin{array}{c}\text { Stationary } \\
\text { phase }\end{array}$ \\
\hline PY22 $\left(\mathrm{LacZ}^{-}\right)$ & $0.8 \pm 0.3$ & $1.3 \pm 1.3$ & $1.3 \pm 0.3$ \\
\hline$r s b R A:: \operatorname{lac} Z$ & $7.1 \pm 1.1$ & $8.4 \pm 2.2$ & $10.1 \pm 1.8$ \\
\hline$r s b R B:: l a c Z$ & $5.9 \pm 1.0$ & $6.2 \pm 0.3$ & $9.5 \pm 1.1$ \\
\hline$r s b R C:: l a c Z$ & $5.4 \pm 0.3$ & $5.9 \pm 0.6$ & $8.4 \pm 0.2$ \\
\hline rsbRD:: lacZ & $1.0 \pm .05$ & $2.4 \pm 0.5$ & $1.6 \pm 0.5$ \\
\hline
\end{tabular}

${ }^{\star}$ Values are means \pm SD of four separate determinations. 
that we employed. The RsbRD levels, although responsive to stress, remained a minor component compared with the other RsbR family members. It is, of course, still possible that there are novel circumstances under which one or more of the $r s b R$ genes is uniquely expressed as the stressosome's principal source of RsbR, but such circumstances do not include the more typical conditions of balanced growth, entry into stationary phase, or the application of common stresses that are known to activate $\sigma^{\mathrm{B}}$. An additional cautionary note is that our results are based on expression of lac $Z$ fusions to the transcriptional and translational regulatory elements of each of the $r s b R$ genes. It is formally possible that there may be undefined posttranslational regulation that could selectively alter the level of a particular RsbR; however, there is no evidence for such regulation at present.

\section{In vivo stressosome formation with single RsbR proteins}

In vitro studies demonstrated that purified RsbRA and its paralogous proteins can form high molecular mass structures that incorporate RsbS (Chen et al., 2003; Delumeau et al., 2006; Kim et al., 2004b). In addition, RsbRB and -C have been detected in stressosome fractions purified from wild-type B. subtilis (Delumeau et al., 2006; Kim et al., 2004b). To examine the degree to which individual RsbR paralogues are able to independently trap RsbS into stressosome complexes in vivo, crude extracts were prepared from wild-type B. subtilis and strains that expressed only one of the paralogues. The extracts were fractionated by velocity centrifugation with the resulting gradient fractions analysed by Western blot using monoclonal antibodies specific for RsbS and, where appropriate, RsbRA. Fig. 1(a) illustrates a representative Western blot obtained from a wild-type B. subtilis strain and below it a quantification of the bands detected by the anti-RsbRA (Fig. 1b) and anti-RsbS (Fig. 1c) antibodies. The coincident peaks of RsbR and RsbS sedimenting between fractions 5 and 10 represent the 'stressosome peak' under these sedimentation conditions. The percentage of the extract's RsbS that migrated to these fractions is presented as the mean obtained from three separate experiments in Table 3. Similar analyses were performed on extracts prepared from strains expressing only one of the RsbR paralogues or a strain missing all four of the RsbR proteins. The antiRsbRA antibody doesn't cross-react with the RsbRB, -RC or -RD proteins. Therefore, only the anti-RsbS antibody could be used in the Western blot assays of these strains. For those extracts that contained one of the RsbR proteins, fast-sedimenting RsbS was observed in the same fractions (5-10), where a peak of RsbR and $-S$ sedimented in Fig. 1(a). The percentage of the RsbS present in these gradient fractions (5-10) is listed in Table 3. The RsbRA extract contained almost as much of its RsbS protein in a fast-sedimenting peak as did the extract from the wild-type strain, which expressed all four of the RsbR proteins (i.e. $53.6 \%$ versus $60 \%$ ). The extract from strains expressing

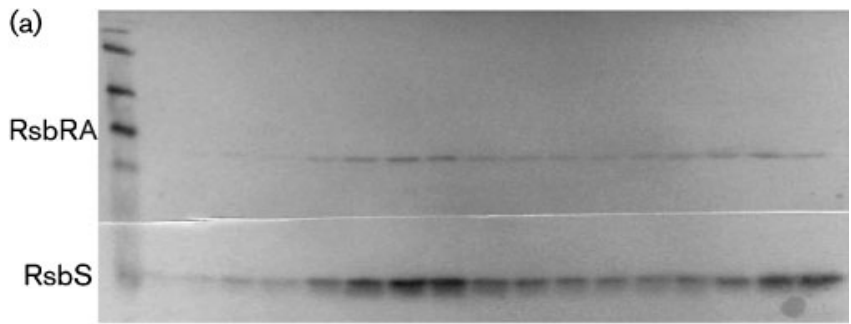

(b)

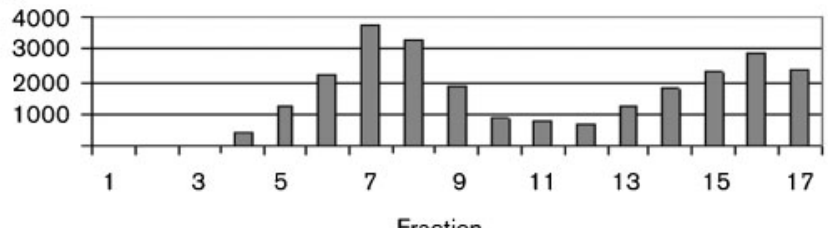

(c)

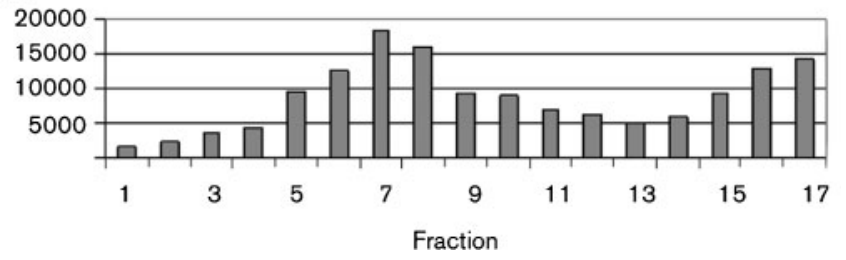

Fig. 1. Sedimentation analysis of RsbRA and RsbS. (a) A crude extract prepared from wild-type B. subtilis (PY22) was layered onto a 5-15\% glycerol gradient and centrifuged for $6 \mathrm{~h}$ at 35000 r.p.m. Fractions were collected and analysed by SDSPAGE and Western blot using monoclonal antibodies specific for RsbRA and RsbS. The positions of RsbRA and RsbS are noted. The net intensity (pixels) of the RsbRA and RsbS bands in each fraction, obtained using a Kodak EDS 290 gel picture station and Kodak 1D.v.3.6.3 software, is presented in (b) and (c), respectively.

single RsbR paralogues also displayed fast-sedimenting RsbS corresponding to positions of stressosome sedimentation, although the percentage of RsbS in these fractions was less than that seen in the wild-type or RsbRA-expressing strain. These differences could be partly due to differences in the expression level of each of the RsbR paralogues. The RsbR protein (RsbRD) with the least amount of RsbS $(14.4 \%)$ in a fast-sedimenting peak is also the paralogue that is expressed at the lowest level (i.e. $<14 \%$ of RsbRA). To explore the possible contribution of expression level on the ability of RsbRD to capture RsbS in a fast-sedimenting complex, the $r s b R D$ sequence was recombined into the B. subtilis chromosome (Methods) as part of the sigB operon, replacing $r s b R A$ and allowing its expression as the cell's sole source of RsbR under the control of the regulatory elements normally responsible for the synthesis of RsbRA. When extracts were prepared from this strain and analysed for RsbS sequestration, the percentage of RsbS in the fast-sedimenting peak rose to $35 \%$ of the RsbS present in the extract (Table 3). Thus, based on the movement of RsbS to fast-sedimenting fractions, each of the RsbR proteins can effectively form a structure that can capture RsbS in vivo. 
Table 3. RsbS distribution of strains expressing a single RsbR paralogue

Crude extracts from indicated strains were subjected to velocity centrifugation and Western blot analyses, as in Fig. 1, using a monoclonal antibody specific for RsbS. After quantification of the RsbS bands, the percentage of the total RsbS that was 'fast sedimenting' (fractions 5-10) was calculated and listed in the table.

\begin{tabular}{|lcc|}
\hline Strain & $\begin{array}{c}\text { Fast sedimenting } \\
\text { RsbS (\%) }\end{array}$ & $\begin{array}{c}\text { Fast-sedimenting RsbS } \\
\text { with RsbR }\end{array}$ \\
\hline PY22 (wild-type) & $60.0 \pm 3.6$ & 54.7 \\
RsbR $^{-}$(BAR299) & $5.3 \pm 2.1$ & \\
RsbRA (BAR298) & $53.6 \pm 7.2$ & 48.3 \\
RsbRB (BAR204) & $36.8 \pm 6.4$ & 31.5 \\
RsbRC (BAR199) & $22.2 \pm 0.4$ & 16.9 \\
RsbRD (BAR205) & $14.4 \pm 3.6$ & 9.1 \\
RsbRD at sigB & $35.3 \pm 2.7$ & 30.0 \\
(BAR308) & & \\
\hline
\end{tabular}

*Values are mean \pm SD of three separate determinations.

A curiosity arising from this analysis is the observation that the percentage of the bacterium's RsbS that is captured by each of the RsbR paralogues into fast-sedimenting complexes is not additive. Individually, each of the RsbRs is able to sequester a percentage of the RsbS that, if added together, would account for all of the cell's RsbS being captured in such complexes. Yet the wild-type strain, in which all of the RsbRs are expressed, acquired only $60 \%$ of the RsbS in such a complex. It is unclear whether this inability to sequester a larger portion of the RsbS represents a manifestation of factors limiting the number of stressosomes that can form at any one time in vivo, a reflection of a balance between stressosome formation and dissociation, or merely an unforeseen consequence of the assay conditions.

\section{RsbT as a limiting component of stressosomes}

It had been reported previously that the expression of any of the phosphorylatable $r s b R s$, including $r s b R D$, is sufficient to control $\sigma^{\mathrm{B}}$ activity during growth (Akbar et al., 2001). Table 4 illustrates $\sigma^{\mathrm{B}}$-dependent reporter gene activity measured during growth and following ethanol induction in wild-type B. subtilis and strains either lacking all of the RsbR proteins or expressing $r s b R A$ or $r s b R D$, or $r s b R D$ from the $r s b R A$ regulatory elements at $\operatorname{sig} B$. As expected, the strain lacking all of the RsbR proteins displays a high level of $\sigma^{\mathrm{B}}$ activity that is not further elevated by stress (Table 4). This unregulated level of $\sigma^{\mathrm{B}}$ activity is somewhat lower than the peak of stress-induced $\sigma^{\mathrm{B}}$ activity and may reflect an adaptive response by the cell to persistently elevated $\sigma^{\mathrm{B}}$ activity. The strain expressing only RsbRD from its own promoter is also effective in controlling $\sigma^{\mathrm{B}}$ activity (Table 4), even though the RsbR protein levels in the strain (Table 2) and the resulting percentage of RsbS in
Table 4. $\sigma^{\mathrm{B}}$ Activity of $r s b R$-expressing strains

B. subtilis expressing the indicated $r s b R$ genes was grown in LB. Samples were taken during growth or at intervals following ethanol addition and assayed for $\sigma^{\mathrm{B}}$-dependent $\beta$-galactosidase activity.

\begin{tabular}{|lcc|}
\hline Strain & \multicolumn{2}{c|}{$\boldsymbol{\beta}$-Galactosidase activity (Miller units) } \\
\cline { 2 - 3 } & Growth $\left(\right.$ OD $\left._{\mathbf{5 4 0}}, \mathbf{0 . 3}-\mathbf{0 . 5}\right)$ & $\begin{array}{c}\text { Ethanol } \\
(\mathbf{4} \%)\end{array}$ \\
\hline BSH80 (wild-type) & $0.8 \pm 0.2$ & $39.8 \pm 1.5$ \\
RsbR $^{-}$(BAR299) & $41.5 \pm 4.5$ & $41.1 \pm 4.0$ \\
RsbRA (BAR298) $_{\text {RsbRD (BAR205) }}$ & $1.6 \pm 0.5$ & $64.1 \pm 3.6$ \\
RsbRD at sigB & $6.8 \pm 2.6$ & $79.5 \pm 5.4$ \\
(BAR308) & $4.1 \pm 0.5$ & $56.7 \pm 0.5$ \\
\hline
\end{tabular}

${ }^{*}$ Values are mean \pm SD of three separate determinations. Miller units taken at the activity peak.

stressosome complexes (Table 3 ) are only a fraction of that seen in the $r s b R A$-expressing strain. The higher level of $\sigma^{\mathrm{B}}$ activity in the unstressed rsbRD-expressing strain, compared to that of the $r s b R A$-expressing strain, is more likely to be a property of the RsbRD protein itself rather than its level of synthesis. We base this notion on the observation that increasing $r s b R D$ expression by placing it under the control of the rsbRA regulatory elements does not appreciably lower $\sigma^{\mathrm{B}}$ activity (Table 4 ), although it significantly raises stressosome abundance (Table 3 ).

Given that the ultimate function of the stressosome is to control the activity of RsbT, the RsbRD strain's ability to hold RsbT's activity in check argues that the lower level of RsbR protein that is present in this strain is sufficient for the proper control of RsbT. This implies that the levels of RsbT in the cell might normally be less than that of the other stressosome components, even though $r s b T$ is cotranscribed with $r s b R A$ and $r s b S$ as part of the $\operatorname{sig} B$ operon (Wise \& Price, 1995). To ask whether RsbRA, -S and $-\mathrm{T}$ are actually synthesized at comparable levels, translational fusions were constructed between the $5^{\prime}$ ends of the $r s b R A, r s b S$ and $r s b T$ genes and E. coli lacZ. When each fusion was integrated into the B. subtilis chromosome and expressed from their normal regulatory elements, comparable levels of $\beta$-galactosidase were measured for each of the translational fusions (Table 5). This result is consistent with RsbT being synthesized at the same rate as RsbRA and RsbS.

We had previously noted that RsbT tended to be more difficult to detect than the other $\operatorname{sig} B$ operon products in Western blots of B. subtilis extracts (Dufour et al., 1996; Kuo et al., 2004). This observation could be a function of the effectiveness of our different antibody preparations, but it could also be an indication that RsbT protein levels are actually lower in B. subtilis than might be expected based on its expression. In an attempt to distinguish between 
Table 5. Translational fusions of lacZ to $r s b R, r s b S$ and $r s b T$

B. subtilis strain PY22 (wild-type), BAR191 (rsbRA:: lacZ), BAR250 ( $r s b S: l a c Z)$, and BAR251 ( $r s b T:: l a c Z)$ were grown to midexponential and stationary phase, where samples were taken and assayed for $\beta$-galactosidase activity.

\begin{tabular}{|lcc|}
\hline \multirow{2}{*}{ Strain } & \multicolumn{2}{c|}{$\boldsymbol{\beta}$-Galactosidase activity (Miller units) } \\
\cline { 2 - 3 } & Growth $\left(\mathbf{O D}_{\mathbf{5 4 0}} \mathbf{0 . 3}-\mathbf{0 . 5}\right)$ & Stationary phase \\
\hline PY22 $\left(\mathrm{LacZ}^{-}\right)$ & $1.0 \pm 0.8$ & $0.6 \pm 0.3$ \\
$r s b R A::$ lacZ & $9.9 \pm 2.5$ & $16.5 \pm 1.90$ \\
rsbS: : lacZ & $9.3 \pm 1.0$ & $12.2 \pm 0.6$ \\
rsbT: : lacZ & $12.3 \pm 1.4$ & $13.1 \pm 1.6$ \\
\hline
\end{tabular}

${ }^{\star}$ Values are mean \pm SD of three separate determinations.

these possibilities, extracts were prepared from an exponentially growing $E$. coli strain expressing $r s b R,-S$ and $-T$ from plasmid pARE7 (Reeves \& Haldenwang, 2007). The relative abundance of RsbRA to RsbT in the E. coli extract was then compared to their relative abundance in similar extracts from vegetatively growing $B$. subtilis (PY22). Fig. 2 illustrates a Western blot assay of these extracts using anti-RsbRA and anti-RsbT antibodies as probes. Due to potential differences in the antibodies' properties, the resulting band intensities do not give a measure of the absolute abundance of each of these proteins, but they can give an indication of the relative abundance of RsbRA to RsbT in each extract. Quantification of the band intensities (Fig. 2) revealed a 10 -fold difference in the ratio of RsbR: RsbT in E. coli relative to that in B. subtilis. Apparently, persistence of RsbT in B. subtilis is restricted relative to RsbRA. It is an open question whether this loss of
RsbT is an inherent property of RsbT or a consequence of its predicted movement between the stressosome and the RsbU phosphatase; however, the observation that the reduced RsbT level is a Bacillus-specific phenomenon suggests the latter.

The data presented in this work argue that it is unlikely that one of the functions of multiple $r s b R$ genes is to provide sources of RsbR under alternative growth conditions. Three ( $r s b R A,-B$ and $-C)$ of the four phosphorylatable $r s b R$ paralogues are expressed at comparable levels, without evident change during growth phase or stress. The fourth paralogue $(r s b R D)$, expressed at low levels during growth, is stress inducible; however, even its induced level allows it to be only a relatively minor component of the total RsbR population. Interestingly, RsbRD is still able to regulate $\sigma^{\mathrm{B}}$ activity even though it sequesters only a small portion of the cell's RsbS into putative stressosomes where the activity of RsbT can be controlled. This is likely to be the result of low RsbT protein levels. Although RsbT is synthesized at the same rate as RsbRA and RsbS, RsbT's ultimate level in growing B. subtilis appears to be only $10 \%$ that of RsbRA.

When the existence of multiple RsbR paralogues was recognized, it was noted that each RsbR provided a different basal level of $\sigma^{\mathrm{B}}$ activity when present as the sole RsbR paralogue in the cell (Akbar et al., 2001). It was unclear whether these differences were a function of the abundance of a given RsbR or its inherent ability to control $\sigma^{\mathrm{B}}$ activity. The current work suggests the latter. RsbRA, $-\mathrm{RB}$ and $-\mathrm{RC}$ are each synthesized at comparable levels (Table 2), and the ability of RsbRD to regulate $\sigma^{\mathrm{B}}$ activity is unchanged even when its apparent abundance is increased threefold (Table 4). Each of the RsbR proteins appears to

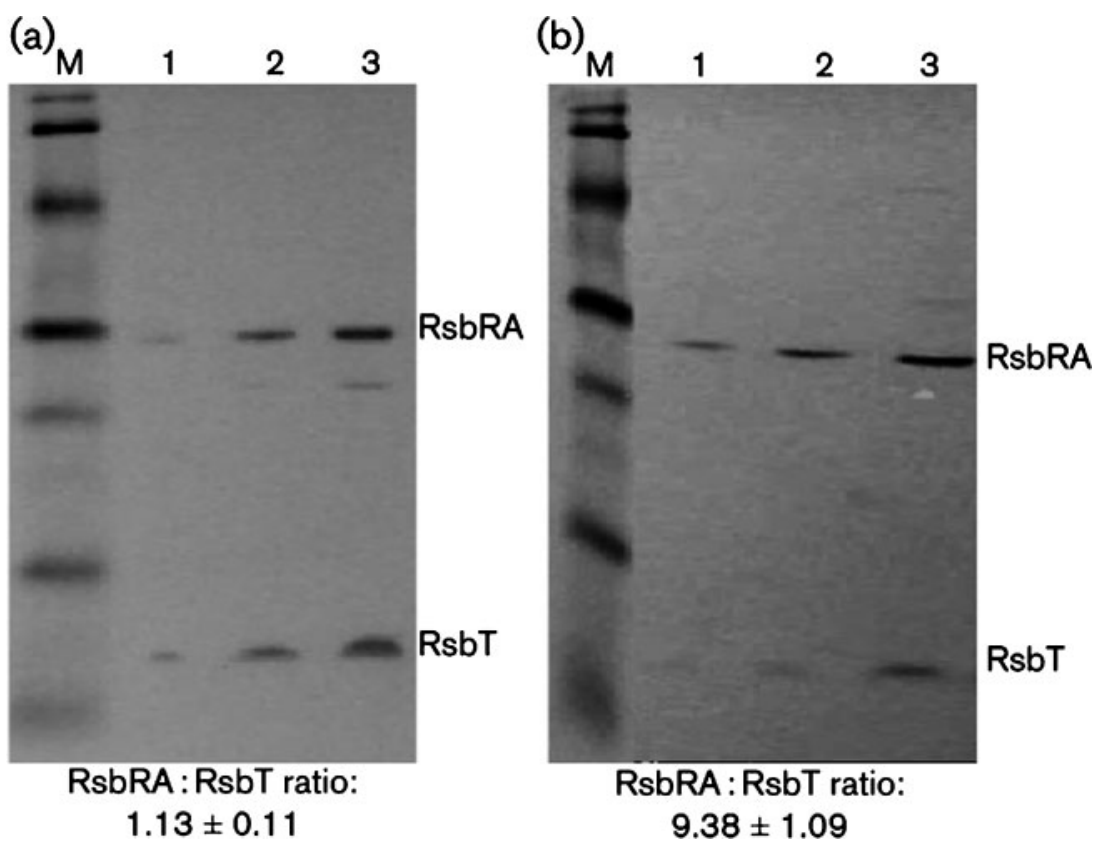

Fig. 2. RsbRA : RsbT ratio in $E$. coli and $B$. subtilis. Crude lysates from (a) E. coli (pARE47) expressing RsbRA, -S, -T and $-\mathrm{U}$, and (b) wild-type $B$. subtilis (PY22) were loaded in twofold increasing amounts (lanes 1-3) onto a $7.5-20 \%$ gradient SDS-PAGE gel. The resulting fractionated proteins were analysed in a common Western blot reaction with monoclonal antibodies specific for RsbRA and RsbT. The Western blot image was captured and the bands were quantified as in Fig. 1. The RsbRA : RsbT ratio for each extract is given below the Western blot images. 
activate $\sigma^{\mathrm{B}}$ in response to a common collection of external stresses. The differences in the degree to which each silences then activates $\sigma^{\mathrm{B}}$ may reflect either its unique biochemical properties (i.e. susceptibility to RsbT phosphorylation, affinity for RsbS binding etc.) or the specific intracellular signal to which it responds. If all of the RsbRs respond to a common trigger, differences in $\sigma^{\mathrm{B}}$ control could reflect distinct activation thresholds for each RsbR. Such a collection of differential responders could allow the stressosome to titre the $\sigma^{\mathrm{B}}$ activation response to the cumulative intensity of the applied stress. Alternatively, a similar effect could be realized if each of the RsbRs does not respond to different levels of the same internal stress signal, but instead to one of multiple internal signals generated by a common external stress. The observation that the N-terminal domains of each of the RsbR proteins, regions believed to be involved in signal recognition, have distinct sequences is more consistent with the notion of multiple signal reception (Marles-Wright et al., 2008). The stressosome would then be an integrator of signals generated by distinct cellular components that are affected by stress. If this is so, it is plausible that each of the putative stress responders, although triggered by most stresses, may not be equally sensitive to all of the stresses that could potentially activate $\sigma^{\mathrm{B}}$. As such, the screening of strains expressing unique subpopulations of RsbRs for their responsiveness to a battery of potential stressors may reveal unique receptivities that could give a clue to the nature of particular responders.

\section{REFERENCES}

Akbar, S., Kang, C. M., Gaidenko, T. A. \& Price, C. W. (1997). Modulator protein RsbR regulates environmental signaling in the general stress pathway of Bacillus subtilis. Mol Microbiol 24, 567578.

Akbar, S., Gaidenko, T. A., Kang, C. M., O'Reilly, M., Devine, K. M. \& Price, C. W. (2001). New family of regulators in the environmental signaling pathway which activates the general stress transcription factor $\sigma^{\mathrm{B}}$ of Bacillus subtilis. J Bacteriol 183, 1329-1338.

Avila-Perez, M., Hellingwerf, K. J. \& Kort, R. (2006). Blue light activates the $\sigma^{\mathrm{B}}$ dependent stress response of Bacillus subtilis via YtvA. $J$ Bacteriol 188, 6411-6414.

Benson, A. K. \& Haldenwang, W. G. (1992). Characterization of a regulatory network that controls $\sigma^{\mathrm{B}}$ expression in Bacillus subtilis. J Bacteriol 174, 749-757.

Benson, A. K. \& Haldenwang, W. G. (1993). Bacillus subtilis $\sigma^{\mathrm{B}}$ is regulated by a binding protein (RsbW) that blocks its association with core RNA polymerase. Proc Natl Acad Sci U S A 90, 2330-2334.

Brody, M. S., Vijay, K. \& Price, C. W. (2001). Catalytic function of an $\alpha /$ $\beta$ hydrolase is responsible for energy stress activation of the $\sigma^{\mathrm{B}}$ transcription factor in Bacillus subtilis. J Bacteriol 183, 6422-6428.

Chen, C.-C., Lewis, R. J., Harris, R., Yudkin, M. D. \& Delumeau, O. (2003). A supermolecular complex in the environmental stress signalling pathway of Bacillus subtilis. Mol Microbiol 49, 1657-1669.

Chen, C.-C., Yudkin, M. D. \& Delumeau, O. (2004). Phosphorylation and RsbX-dependent dephosphorylation of RsbR in the RsbR-RsbS complex of Bacillus subtilis. J Bacteriol 186, 6830-6836.
Delumeau, O., Lewis, R. J. \& Yudkin, M. D. (2002). Protein-protein interactions that regulate the energy stress activation of $\sigma^{\mathrm{B}}$ in Bacillus subtilis. J Bacteriol 184, 5583-5589.

Delumeau, O., Chen, C.-C., Murray, J. W., Yudkin, M. D. \& Lewis, R. J. (2006). High molecular weight complexes of RsbR and paralogues in the environmental signaling pathway. J Bacteriol 188, 78857892.

Dufour, A. \& Haldenwang, W. G. (1994). Interactions between a Bacillus subtilis anti- $\sigma$ factor (RsbW) and its antagonist (RsbV). J Bacteriol 176, 1813-1820.

Dufour, A., Voelker, U., Voelker, A. \& Haldenwang, W. G. (1996). Relative levels and fractionation properties of Bacillus subtilis $\sigma^{\mathrm{B}}$ and its regulators during balanced growth and stress. J Bacteriol 178, 3701-3709.

Errington, J. (1986). A general method for fusion of the Escherichia coli lacZ gene to chromosomal genes in Bacillus subtilis. J Gen Microbiol 132, 2953-2966.

Eymann, C., Becher, D., Bernhardt, J., Gronau, K., Klutzny, A. \& Hecker, M. (2007). Dynamics of protein phosphorylation on Ser/Thr/ Tyr in Bacillus subtilis. Proteomics 7, 3509-3526.

Gaidenko, T. A., Yang, X., Lee, Y. M. \& Price, C. W. (1999). Threonine phosphorylation of modulator protein RsbR governs its ability to regulate a serine kinase in the stress signaling pathway of Bacillus subtilis. J Mol Biol 288, 29-39.

Gaidenko, T. A., Kim, T.-J., Weigel, A. L., Brody, M. S. \& Price, C. W. (2006). The blue-light receptor YtvA acts as an environmental stress signaling pathway of Bacillus subtilis. J Bacteriol 188, 63876395.

Guérout-Fleury, A.-M., Shazand, K., Frandsen, N. \& Stragier, P. (1995). Antibiotic-resistance cassettes for Bacillus subtilis. Gene 167, 335-336.

Hardwick, S. W., Pane-Farre, J., Delumeau, O., Marles-Wright, J., Murray, J. W., Hecker, M. \& Lewis, R. (2007). Structural and functional characterization of partner switching regulating the environmental stress response in Bacillus subtilis. J Biol Chem 282, 11562-11572.

Hecker, M., Schumann, W. \& Voelker, U. (1996). Heat-shock and general stress response in Bacillus subtilis. Mol Microbiol 19, 417-428.

Hecker, M., Pane-Farre, J. \& Volker, U. (2007). SigB-dependent general stress response in Bacillus subtilis and related Gram-positive bacteria. Annu Rev Microbiol 61, 215-236.

Jonas, R. M., Peters, H. K., III \& Haldenwang, W. G. (1990). Phenotypes of Bacillus subtilis mutants altered in the precursor-

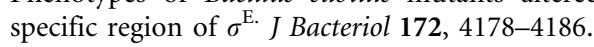

Kang, C. M., Brody, M. S., Akbar, S., Yang, X. \& Price, C. W. (1996). Homologous pairs of regulatory proteins control activity of Bacillus subtilis transcription factor $\sigma^{\mathrm{B}}$ in response to environmental stress. J Bacteriol 178, 3846-3853.

Kenney, T. J. \& Moran, C. P., Jr (1987). Organization and regulation of an operon that encodes a sporulation-essential sigma factor of Bacillus subtilis. J Bacteriol 169, 3329-3339.

Kim, T.-J., Gaidenko, T. A. \& Price, C. W. (2004a). In vivo phosphorylation of partner switching regulators correlates with stress transmission in the environmental signaling pathway of Bacillus subtilis. J Bacteriol 186, 6124-6132.

Kim, T.-J., Gaidenko, T. A. \& Price, C. W. (2004b). A multi-component protein complex mediates environmental stress signaling in Bacillus subtilis. J Mol Biol 341, 135-150.

Kuo, S., Zhang, S., Woodbury, R. L. \& Haldenwang, W. G. (2004). Associations between Bacillus subtilis $\sigma^{\mathrm{B}}$ regulators in cell extracts. Microbiology 150, 4125-4136. 
Marles-Wright, J., Grant, T., Delumeau, O., van Duinin, G., Firbank, S. J., Lewis, P. J., Murray, J. W., Newman, J. A., Quin, M. B. \& other authors (2008). Molecular architecture of the "stressosome," a signal integration and transduction hub. Science 322, 92-96.

Petersohn, A., Bernhardt, J., Gerth, U., Hoper, D., Koburger, T., Voelker, U. \& Hecker, M. (1999). Identification of $\sigma^{\mathrm{B}}$-dependent genes in Bacillus subtilis using a promoter consensus-directed search and oligonucleotide hybridization. J Bacteriol 181, 5718-5724.

Price, C. W., Fawcett, P., Ceremonie, H., Su, N., Murphy, C. K. \& Youngman, P. (2001). Genome-wide analysis of the general stress response in Bacillus subtilis. Mol Microbiol 41, 757-774.

Reeves, A. \& Haldenwang, W. G. (2007). Isolation and characterization of dominant mutations in the Bacillus subtilis stressosome components RsbR and RsbS. J Bacteriol 189, 1531-1541.

Reeves, A., Gerth, U., Volker, U. \& Haldenwang, W. G. (2007). ClpP modulates the activity of Bacillus subtilis stress response transcription factor $\sigma^{\mathrm{B}}$. J Bacteriol 189, 6168-6175.

Sambrook, J., Fritsch, E. F. \& Maniatis, T. (1989). Molecular Cloning: a Laboratory Manual, 2nd edn. Cold Spring Harbor, NY: Cold Spring Harbor Laboratory.

Suzuki, N., Takaya, N., Hashino, T. \& Nakamura, A. (2007). Enhancement of a $\sigma^{\mathrm{B}}$-dependent stress response in Bacillus subtilis by light via the YtvA photoreceptor. J Gen Appl Microbiol 53, 81-88.

Vijay, K., Brody, M. S., Fredlund, E. \& Price, C. W. (2000). A PP2C phosphatase containing a PAS domain is required to convey signals of energy stress to the $\sigma^{\mathrm{B}}$ transcription factor of Bacillus subtilis. Mol Microbiol 35, 180-188.
Voelker, U., Voelker, A., Maul, B., Hecker, M., Dufour, A. \& Haldenwang, W. G. (1995). Separate mechanisms activate $\sigma^{\mathrm{B}}$ of Bacillus subtilis in response to environmental and metabolic stresses. J Bacteriol 177, 3771-3780.

Voelker, U., Voelker, A. \& Haldenwang, W. G. (1996). Reactivation of the Bacillus subtilis anti- $\sigma^{\mathrm{B}}$ antagonist, RsbV, by stress or starvation-induced phosphatase activities. J Bacteriol 178, 54565463.

Voelker, U., Luo, T., Smirnova, N. \& Haldenwang, W. G. (1997). Stress activation of Bacillus subtilis $\sigma^{\mathrm{B}}$ can occur in the absence of the $\sigma^{\mathrm{B}}$ negative regulator RsbX. J Bacteriol 179, 1980-1984.

Wise, A. A. \& Price, C. W. (1995). Four additional genes in the sigB operon of Bacillus subtilis that control activity of the general stress factor $\sigma^{\mathrm{B}}$ in response to environmental signals. J Bacteriol 177, 123133.

Yang, X., Kang, C. M., Brody, M. S. \& Price, C. W. (1996). Opposing pairs of serine protein kinases and phosphatases transmit signals of environmental stress to activate a bacterial transcription factor. Genes Dev 10, 2265-2275.

Yasbin, R. E., Wilson, G. A. \& Young, F. E. (1973). Transformation and transfection of lysogenic strains of Bacillus subtilis 168. J Bacteriol 113, 540-548.

Zhang, S. \& Haldenwang, W. G. (2005). Contributions of ATP, GTP, and redox state to nutritional stress activation of the Bacillus subtilis $\sigma^{\mathrm{B}}$ transcription factor. J Bacteriol 187, 7554-7560.

Edited by: C. W. Price 